\title{
On the associated primes of generalized local cohomology modules
}

\author{
Amir Mafi * \\ Institute of Mathematics, University for Teacher Education, 599 Taleghani \\ Avenue, Tehran 15614, Tehran Iran-and-Department of Mathematic, Arak \\ University, Arak, Iran
}

\section{INTRODUCTION}

Throughout this paper, $R$ is a commutative Noetherian ring with identity. For an ideal $\mathfrak{a}$ of $R$ and $i \geq 0$, the $i$-th local cohomology module of $M$ is defined as:

$$
H_{\mathfrak{a}}^{i}(M)=\underset{n}{\lim } \operatorname{Ext}_{R}^{i}\left(R / \mathfrak{a}^{n}, M\right)
$$

In [8], Huneke conjectured that if $M$ is a finitely generated $R$-module, then the set of associated primes of $H_{\mathfrak{a}}^{i}(M)$ is finite. Singh [15] provides a counter example for this conjecture. However, it is known that the conjecture is true in many situations. For example, in [11] it is shown that if $R$ is local and $\operatorname{dim} R / \mathfrak{a}=1$, then for a finitely generated $R$-module $M$, the set $\operatorname{Ass}_{R}\left(H_{\mathfrak{a}}^{i}(M)\right)$ is finite for all $i \geq 0$.

Also, Brodmann and Lashgari [2] showed that the first non-finitely generated local cohomology module of a finitely generated $R$-module has only finitely many associated primes. Also, see [10] and [4] for a far reaching generalizations of this result.

The following generalization of local cohomology theory is due to Herzog [7] (see also [17]). The generalized local cohomology functor $H_{\mathfrak{a}}^{i}(.,$.$) is defined by$

$$
H_{\mathfrak{a}}^{i}(M, N)=\underset{n}{\lim } \operatorname{Ext}_{R}^{i}\left(M / \mathfrak{a}^{n} M, N\right)
$$

for all $R$-modules $M$ and $N$. Clearly, this is a generalization of the usual local cohomology functor. Recently, there are some new interest in generalized local cohomology (see e.g. [1], [5], [6] and [18]). Our main aim in this paper is to establish the following.

Theorem 1.1. Let $\mathfrak{a}$ be an ideal of $R$ and let $M$ and $N$ be two finitely generated $R$-modules. Then the following statements hold. 
(i) For any positive integer $t$,

$$
\operatorname{Ass}_{R}\left(H_{\mathfrak{a}}^{t}(M, N)\right) \subseteq \bigcup_{i=0}^{t} \operatorname{Ass}_{R}\left(\operatorname{Ext}_{R}^{i}\left(M, H_{\mathfrak{a}}^{t-i}(N)\right)\right)
$$

(ii) If $d=p d(M)$ and $n=\operatorname{dim} N$ are finite, then $H_{\mathfrak{a}}^{n+d}(M, N)$ is Artinian. In particular $\operatorname{Ass}_{R}\left(H_{\mathfrak{a}}^{n+d}(M, N)\right)$ consists of finitely many maximal ideals.

(iii) Suppose that $(R, \mathfrak{m})$ is local with dimension $n$ and that $d=p d(M)$ is finite. Then $\operatorname{Supp}_{R}\left(H_{\mathfrak{a}}^{n+d-1}(M, N)\right)$ is finite.

Clearly (i) extends the main results of $[\mathbf{1 0}$, Theorem B], $[\mathbf{2}$, Theorem 2.2] and $[\mathbf{4}$, Corollary 2.7], (ii) extends [12, Theorem 2.2], and (iii) is an improvement of [11, Corollary 2.4].

\section{The Results}

First, we recall the definition of a weakly Laskerian module. An $R$-module $M$ is said to be Laskerian if any submodule of $M$ is an intersection of a finite number of primary submodules. Obviously, any Noetherian module is Laskerian. In [4], as a generalization of this notion, we introduced the following definition.

Definition 2.1. An $R$-module $M$ is said to be weakly Laskerian if the set of associated primes of any quotient module of $M$ is finite.

Example 2.2. (i) Every Laskerian module is weakly Laskerian.

(ii) Any module with finite support is weakly Laskerian. In particular, any Artinian $R$-module is weakly Laskerian.

Theorem 2.3. Let $\mathfrak{a}$ be an ideal of $R$ and $M$ be a finitely generated $R$-module. Let $N$ be an $R$-module and $t$ a positive integer. Then

$$
\operatorname{Ass}_{R}\left(H_{\mathfrak{a}}^{t}(M, N)\right) \subseteq \bigcup_{i=0}^{t} \operatorname{Ass}_{R}\left(\operatorname{Ext}_{R}^{i}\left(M, H_{\mathfrak{a}}^{t-i}(N)\right)\right)
$$

Proof. By [14, Theorem 11.38], there is a Grothendieck spectral sequence

$$
E_{2}^{p, q}:=\operatorname{Ext}_{R}^{p}\left(M, H_{\mathfrak{a}}^{q}(N)\right) \underset{p}{\Longrightarrow} H_{\mathfrak{a}}^{p+q}(M, N) .
$$

For all $i \geq 2$, we consider the exact sequence

$$
0 \longrightarrow \operatorname{ker} d_{i}^{0, t} \longrightarrow E_{i}^{0, t} \stackrel{d_{i}^{0, t}}{\longrightarrow} E_{i}^{i, t-i+1} \cdot(1)
$$


Since $E_{i}^{0, t}=\operatorname{ker} d_{i-1}^{0, t} / \operatorname{im} d_{i-1}^{1-i, t+i-2}$ and $E_{i}^{i, j}=0$ for all $j<0$, we may use (1) to obtain $\operatorname{ker} d_{t+2}^{i, t-i} \cong E_{t+2}^{i, t-i} \cong \cdots=E_{\infty}^{i, t-i}$ for all $0 \leq i \leq t$. There exists a finite filtration

$$
0=\phi^{t+1} H^{t} \subseteq \phi^{t} H^{t} \subseteq \cdots \subseteq \phi^{1} H^{t} \subseteq \phi^{0} H^{t}=H_{\mathfrak{a}}^{t}(M, N)
$$

such that

$$
E_{\infty}^{i, t-i}=\phi^{i} H^{t} / \phi^{i+1} H^{t}
$$

for all $0 \leq i \leq t$

Now, the exact sequences $0 \longrightarrow \phi^{i+1} H^{t} \longrightarrow \phi^{i} H^{t} \longrightarrow E_{\infty}^{i, t-i} \longrightarrow 0(0 \leq i \leq t)$ in conjunction with

$$
E_{\infty}^{i, t-i} \cong \operatorname{ker} d_{t+2}^{i, t-i} \subseteq \operatorname{ker} d_{2}^{i, t-i} \subseteq E_{2}^{i, t-i}
$$

yields

$$
\operatorname{Ass}_{R}\left(H_{\mathfrak{a}}^{t}(M, N)\right) \subseteq \bigcup_{i=0}^{t} \operatorname{Ass}_{R}\left(\operatorname{Ext}_{R}^{i}\left(M, H_{\mathfrak{a}}^{t-i}(N)\right)\right) . \square
$$

Next, we obtain an extension of $[\mathbf{2}$, Theorem 2.2], [10, Theorem B], and $[\mathbf{4}$, Corollary 2.7].

Corollary 2.4. Let $\mathfrak{a}$ be an ideal of $R, M$ a finitely generated $R$-module, and $N a$ weakly Laskerian $R$-module. If $H_{\mathfrak{a}}^{i}(N)$ is weakly Laskerian module for all $i<t$, then $\operatorname{Ass}_{R}\left(H_{\mathfrak{a}}^{t}(M, N)\right)$ is finite.

Proof. This is immediate by 2.3 and [4, Lemma 2.3 and Corollary 2.7].

Corollary 2.5. Let $(R, \mathfrak{m})$ be a local ring and let $\operatorname{dim} R \leq 2$. Let $M$ be a finitely generated $R$-module and $N$ a weakly Laskerian $R$-module. Then $\operatorname{Ass}_{R}\left(H_{\mathfrak{a}}^{t}(M, N)\right.$ is finite for all $t \geq 0$.

Proof. By [11, Corollaries 2.3, 2.4], [3, Theorem 6.1.2] and 2.2(ii), $H_{\mathfrak{a}}^{t}(N)$ is weakly Laskerian for all $t \geq 1$. Also, $\Gamma_{\mathfrak{a}}(N)$ is weakly Laskerian by 2.1 . So, by 2.4 , $\operatorname{Ass}_{R}\left(H_{\mathfrak{a}}^{t}(M, N)\right)$ is finite for all $t \geq 0$.

Corollary 2.6. Let $\mathfrak{a}$ be an ideal of a local ring $(R, \mathfrak{m})$ and $\operatorname{dim} R=n$. Let $M$ be a finitely generated $R$-module and $N$ be an $R$-module such that $H_{\mathfrak{a}}^{i}(N)=0$ for all $i \neq n-1, n$. Then $\operatorname{Ass}_{R}\left(H_{\mathfrak{a}}^{t}(M, N)\right)$ is finite for all $t \geq 0$.

Proof. By [11, Corollaries 2.3, 2.4] and hypothesis, $\operatorname{Supp}_{R}\left(H_{\mathfrak{a}}^{t}(N)\right)$ is finite for all $t \geq 0$; so that, by $2.4, \operatorname{Ass}_{R}\left(H_{\mathfrak{a}}^{t}(M, N)\right)$ is finite for all $t \geq 0$. 
Corollary 2.7. Let $\mathfrak{a}$ be an ideal of a local ring $(R, \mathfrak{m})$ with $\operatorname{dim} R / \mathfrak{a}=1$. Let $M$ and $N$ be two $R$-modules. Then $\operatorname{Ass}_{R}\left(H_{\mathfrak{a}}^{t}(M, N)\right)$ is finite for all $t \geq 0$.

Proof. It is clear that $\operatorname{Supp}_{R}\left(H_{\mathfrak{a}}^{t}(N)\right)$ is finite for all $t \geq 0$; hence by 2.4, $\operatorname{Ass}_{R}\left(H_{\mathfrak{a}}^{t}(M, N)\right)$ is finite for all $t \geq 0$.

Following $[\mathbf{9}]$, a sequence $x_{1}, \ldots, x_{n}$ of elements of $\mathfrak{a}$ is said to be an $\mathfrak{a}$-filter regular sequence on $N$, if

$$
\operatorname{Supp}_{R}\left(\left(x_{1}, \ldots, x_{i-1}\right) N:_{N} x_{i} /\left(x_{1}, \ldots, x_{i-1}\right) N\right) \subseteq V(\mathfrak{a})
$$

for all $i=1, \ldots, n$, where $V(\mathfrak{a})$ denotes the set of all prime ideals of $R$ containing $\mathfrak{a}$. The concept of an $\mathfrak{a}$-filter regular sequence is a generalization of the one of a filter regular sequence which has been studied in [16, Appendix 2(ii)] and has led to some interesting results. It is easy to see that the analogue of [16, Appendix 2(ii)] holds true whenever $R$ is Noetherian, $N$ is a finitely generated $R$-module and $\mathfrak{m}$ replaced by $\mathfrak{a}$; so that, if $x_{1}, \ldots x_{n}$ be an $\mathfrak{a}$-filter regular sequence on $N$, then there is an element $y \in \mathfrak{a}$ such that $x_{1}, \ldots, x_{n}, y$ is an $\mathfrak{a}$-filter regular sequence on $N$. Thus for a positive integer $n$, there exists an $\mathfrak{a}$-filter regular sequence on $N$ of length $\mathrm{n}$.

The following Lemma, which needs the concept of a filter regular sequence, is a generalization of [13, Lemma 3.4].

Lemma 2.8. Let $\mathfrak{a}$ be an ideal of $R$ and $M$ be a finitely generated $R$-module such that $d=p d(M)$ is finite. Let $N$ be an $R$-module and assume that $n \in \mathbb{N}$ and $x_{1}, \ldots, x_{n}$ is an $\mathfrak{a}$-filter regular sequence on $N$. Then $H_{\mathfrak{a}}^{i+n}(M, N) \cong H_{\mathfrak{a}}^{i}\left(M, H_{\left(x_{1}, \ldots, x_{n}\right)}^{n}(N)\right)$ for all $i \geq d$.

Proof. Consider the spectral sequence

$$
E_{2}^{p, q}:=H_{\mathfrak{a}}^{p}\left(M, H_{\left(x_{1}, \ldots, x_{n}\right)}^{q}(N)\right) \underset{p}{\Longrightarrow} H_{\mathfrak{a}}^{p+q}(M, N) .
$$

We have $E_{2}^{p, q}=0$ for $q>n$ (by Theorem 3.3.1 of [3]) and for $q=n, p>d$ (by Proposition 2.5 of [13] and Lemma 1.1 of [18]). It therefore follows $E_{2}^{i, n} \cong E_{\infty}^{i, n}$ and $E_{\infty}^{i, n} \cong H_{\mathfrak{a}}^{i+n}(M, N)$. This proves the result.

The following result is a generalization of [12, Theorem 2.2] and [6, Theorem 1.2]. 
Theorem 2.9. Let $\mathfrak{a}$ be an ideal of $R$ and let $M$ and $N$ be two finitely generated $R$-modules. Assume that $d=\operatorname{pd}(M)$ and $n=\operatorname{dim} N$ are finite. Then $H_{\mathfrak{a}}^{n+d}(M, N)$ is an Artinian $R$-module. In particular, $\operatorname{Ass}_{R}\left(H_{\mathfrak{a}}^{n+d}(M, N)\right.$ is a finite set consisting of maximal ideals.

Proof. Let $x_{1}, \ldots, x_{n}$ be an $\mathfrak{a}$-filter regular sequence on $N$. Then, by 2.8 ,

$$
H_{\mathfrak{a}}^{n+d}(M, N) \cong H_{\mathfrak{a}}^{d}\left(M, H_{\left(x_{1}, \ldots, x_{n}\right)}^{n}(N)\right)
$$

and, by [3, Exercise 7.1.7], $H_{\left(x_{1}, \ldots, x_{n}\right)}^{n}(N)$ is Artinian. Put $S=H_{\left(x_{1}, \ldots, x_{n}\right)}^{n}(N)$. Then $H_{\mathfrak{a}}^{d}(M, S) \cong H^{d}\left(\operatorname{Hom}\left(M, \Gamma_{\mathfrak{a}}(\dot{E})\right)\right)$ by $[\mathbf{6}$, Lemma 2.1$]$, where $\dot{E}$ is an injective resolution of $S$ such that its terms are all Artinian modules. Therefore $H_{\mathfrak{a}}^{n+d}(M, N)$ is Artinian and $\operatorname{Ass}_{R}\left(H_{\mathfrak{a}}^{n+d}(M, N)\right)$ is a finite set consisting of maximal ideals.

The following theorem is an improvement of [11, Corollary 2.4].

Theorem 2.10. Let $(R, \mathfrak{m})$ be a local ring of dimension $n, N$ an $R$-module, $M a$ finitely generated $R$-module, and $d=p d(M)$ is finite. Then $\operatorname{Supp}_{R}\left(H_{\mathfrak{a}}^{n+d-1}(M, N)\right)$ is finite.

Proof. Consider the Grothendieck spectral sequence

$$
E_{2}^{p, q}:=\operatorname{Ext}_{R}^{p}\left(M, H_{\mathfrak{a}}^{q}(N)\right) \underset{p}{\Longrightarrow} H_{\mathfrak{a}}^{p+q}(M, N) .
$$

So, we have a finite filtration

$$
0=\phi^{d+n} H^{d+n-1} \subseteq \phi^{d+n-1} H^{d+n-1} \subseteq \cdots \subseteq \phi^{1} H^{d+n-1} \subseteq \phi^{0} H^{d+n-1}=H_{\mathfrak{a}}^{d+n-1}(M, N)
$$

and the equalities $E_{\infty}^{i, d+n-i-1}=\phi^{i} H^{n+d-1} / \phi^{i+1} H^{n+d-1}$ for all $o \leq i \leq n+d-1$.

Since $E_{2}^{i, n+d-i-1}=0$ for all $i \neq d-1, d$ and $E_{\infty}^{i, n+d-i-1}$ is a subquotient $E_{2}^{i, n+d-i-1}$, it follows that

$$
\phi^{d+1} H^{n+d-1}=\phi^{d+2} H^{n+d-1}=\ldots=\phi^{d+n} H^{n+d-1}=0
$$

and that

$$
\phi^{d-1} H^{n+d-1}=\phi^{d-2} H^{n+d-1}=\ldots=\phi^{0} H^{n+d-1}=H_{\mathfrak{a}}^{n+d-1}(M, N) .
$$

Now, using the above consequences in conjunction with [11, Corollaries 2.3, 2.4], it is easy to see that $\operatorname{Supp}_{R}\left(E_{\infty}^{d, n-1}\right)$ and $\operatorname{Supp}_{R}\left(E_{\infty}^{d-1, n}\right)$ are finite sets.

Next, consider the exact sequence

$$
0 \longrightarrow E_{\infty}^{d, n-1} \longrightarrow H_{\mathfrak{a}}^{n+d-1}(M, N) \longrightarrow E_{\infty}^{d-1, n} \longrightarrow 0
$$


to deduce that $\operatorname{Supp}_{R}\left(H_{\mathfrak{a}}^{n+d-1}(M, N)\right)$ is a finite.

\section{REFERENCES}

[1] J. Asadollahi, K. Khashayarmanesh and Sh. Salarian, On the finiteness properties of the generalized local cohomology modules, Comm. Algebra., 30(2)(2002), 859-867.

[2] M. P. Brodmann and F. A. Lashgari, A finiteness result for associated primes of local cohomology modules, Proc. Amer. Math. Soc., 128(10)(2000), 2851-2853.

[3] M. P. Brodmann and R. Y. Sharp, Local cohomology-An algebric introduction with geometric applications , Cambr. Uni. Press, 1998.

[4] K. Divaani-Aazar and A. Mafi, Associated prime of local cohomology modules, Proc. Amer. Math. Soc., to appear.

[5] K. Divaani-Aazar and R. Sazeedeh, Cofiniteness of generalized local cohomology modules, Colloquium Mathematiqum, to appear.

[6] K. Divaani-Aazar, R. Sazeedeh and M. Tousi, On vanishing of generalized local cohomology modules, Algebra Colloquium, to appear.

[7] J. Herzog, Komplexe, Auflösungen und dualitat in der localen Algebra, Habilitationss chrift, Universität Regensburg. 1970.

[8] C. Huneke, Problems on local cohomology, Free resolutions in commutative algebra and algebraic geometry, Res. Notes Math., 2 (1992), 93-108.

[9] K. Khashyarmanesh and Sh. Salarian, Filter regular sequences and the finitness of local cohomology modules, Comm. Algebra, 26(8)(1998), 2483-2490.

[10] K. Khashyarmanesh and Sh. Salarian, On the associated primes of local cohomology modules, Comm. Algebra, 27(12) (1999), 6191-6198.

[11] T. Marley, The associated primes of local cohomology modules of small dimension, Manuscripta Math. 104(4)(2001), 519-525.

[12] L. Melkersson, Some application of criterion for Artinianness of a module, J. Pure. Appl. Algebra., 101 (1995), 291-303.

[13] U. Nagel and P. Schenzel, Cohomological annihilators and Castelnuovo mumford regularity, Commutative algebra: Syzygies, Multiplicities, and birational algebra, (South Hadley, MA , (1992), 307-328). Contemp. Math. Providence, RI(1994).

[14] J. Rotman, Introduction to homological algebra, Academic Press, 1979.

[15] A.K. Singh, P-torsion elements in local cohomology modules, Math. Res. Lett., 7(2-3) (2000), 165-176.

[16] J. Stückrad and W. Vogel, Buchsbaum rings and applications, VEB Deutscher Verlag der wissenschaftan, Berlin(1986).

[17] N. Suzuki, On the generalized local cohomology and its duality, J. Math. Kyoto University, 18(1978), 71-85.

[18] S. Yassemi, L. Khatami and T. Sharif, Associated primes of generalized local cohomology modules, Comm. Algebra, 30(1)(2002), 327-330. 